OPEN ACCESS

Edited by:

Emma Losito,

Hôpital Necker-Enfants Malades,

APHP, France

Reviewed by:

Yam Nath Paudel,

Monash University Malaysia, Malaysia Daichi Sone,

University College London,

United Kingdom

${ }^{*}$ Correspondence:

Weihong Lin

linwh@jlu.edu.cn

Specialty section:

This article was submitted to

Epilepsy,

a section of the journal

Frontiers in Neurology

Received: 27 July 2021 Accepted: 06 October 2021 Published: 28 October 2021

Citation:

Li N, Li J, Chen Y, Chu C and Lin W (2021) Treatment Outcome and Risk

Factors of Adult Newly Diagnosed

Epilepsy: A Prospective

Hospital-Based Study in Northeast

China. Front. Neurol. 12:747958.

doi: 10.3389/fneur.2021.747958

\section{Treatment Outcome and Risk Factors of Adult Newly Diagnosed Epilepsy: A Prospective Hospital-Based Study in Northeast China}

\author{
Nan $\mathrm{Li}^{1}$, Jing $\mathrm{Li}^{1}$, Yanyan Chen ${ }^{1,2}$, Chaojia Chu ${ }^{1}$ and Weihong $\mathrm{Lin}^{1 *}$ \\ ${ }^{1}$ Department of Neurology, The First Hospital of Jilin University, Changchun, China, ${ }^{2}$ Department of Neuroelectrophysiology, \\ Changchun Six Hospital, Changchun, China
}

Objective: The study was conducted to summarize the treatment outcomes of newly diagnosed epilepsy (NDE) and analyse the risk factors for refractory epilepsy (RE) in Northeast China.

Methods: A total of 466 adult patients with NDE were consecutively enrolled in this programme. Clinical data were collected at baseline and each follow-up. Several scales concerning recognition and mood were also completed at the first visit.

Results: Seizure-free status was achieved by $52 \%(n=244)$ of the patients; however, $15 \%(n=68)$ manifested RE. A total of $286(61 \%)$ patients continued with the first ASM as monotherapy, among which 186 (40\%) patients became seizure-free. Fifteen (22\%) patients with RE became seizure-free following ASM adjustment and 34 patients (14\%) had breakthrough seizures after being classified as seizure-free. One patient developed $\mathrm{RE}$ after attaining seizure-free status. Breakthrough seizures during the first expected interictal interval [Odds ratio $(\mathrm{OR})=5.81,95 \% \mathrm{Cl}$ : $2.70-12.50]$, high seizure frequency at baseline (OR $=1.24,95 \% \mathrm{Cl}: 1.04-1.49)$, younger age of onset $(\mathrm{OR}=1.42,95 \% \mathrm{Cl}$ : 1.12-1.79), and male sex (OR $=2.64,95 \% \mathrm{Cl}$ : $1.26-5.53)$ were risk factors for $\mathrm{RE}$.

Significance: Treatment outcomes of the majority of NDE cases are good. New risk factors could help physicians more promptly and accurately identify patients who are likely to develop RE. Seizure-free state is not long enough to commence the withdrawal of ASMs. RE is not permanent and seizure-free may be achieved subsequently by appropriate drug adjustment.

Keywords: drug resistant epilepsy, antiseizure medication, risk factors, adult, newly diagnosed epilepsy

\section{INTRODUCTION}

Epilepsy is a serious neurological disorder that affects more than 70 million people worldwide, ranging from neonates to older adults (1). In China, the number of patients with epilepsy was $\sim 10$ million in 2015 (2). Pharmacotherapy is the first choice for controlling epileptic seizures, and the majority of them could be controlled by currently available antiseizure medication (ASM). Refractory epilepsy (RE) is one of the most serious conditions, which affects $30-40 \%$ of people with epilepsy $(3,4)$. After years of multi-drug treatment with limited efficacy, patients with RE face great financial burden and mental pressure that seriously affect 
their quality of life. In this situation, making a precise diagnosis of $\mathrm{RE}$ is critical and would give a chance for appropriate subsequent treatments, such as neurostimulation and surgery. In previous studies (5-7), the diagnostic criteria for RE were inconsistent; thus, it is difficult to compare the conclusions across them. To set up explicit and practical criteria, the International League Against Epilepsy (ILAE) published a new definition of RE (8). That is, the minimum criteria for defining RE, ensuring that less time was wasted in inappropriate pharmacological therapy, thereby improving patient care. However, the definition has not been widely applied to the epidemiologic studies. Finding risk factors according to the new definition could help the physicians more promptly and accurately identify patients who are likely to develop RE.

This study consecutively enrolled patients with newly diagnosed epilepsy (NDE) at the Epilepsy Diagnosis and Treatment Center of the First Hospital of Jilin University, which is one of the biggest general hospitals in Jilin province, China. We summarized the treatment outcomes of NDE and analyzed the risk factors of RE in Northeast China.

\section{MATERIALS AND METHODS}

\section{Patient Recruitment}

Patients visiting the Epilepsy Diagnosis and Treatment Center of the First Hospital of Jilin University were screened, and the adult patients who were newly diagnosed with epilepsy were consecutively enrolled in this programme between June 2015 and November 2019, and followed up until December 2020.

The definitions of epilepsy, the classification of seizure, and epileptic syndrome conformed to the diagnostic criteria published by ILAE (9-11). RE is defined as the failure of two tolerated and appropriate ASMs (whether monotherapy or in combination) to achieve sustained seizure-free state (8). The $50 \%$ defined daily dose (50\% DDD) is considered as the "adequate dose" of each ASM (12). When patients are free from all seizures, including aura, for three times the interictal interval or 1 year (whichever is longer), they can be classified as seizure-free $(8,13)$. If the two abovementioned definitions cannot be satisfied, the outcome is designated as undetermined. The definition of a patient with NDE used in this study is a person with confirmed epilepsy who had not been diagnosed specifically with epilepsy or treated with ASMs previously.

\section{Study Procedure}

At their first visit, all the participants underwent a thorough clinical and laboratory investigation, including a $24-\mathrm{h}$ video electroencephalogram (EEG) and 3.0-T high-resolution brain magnetic resonance imaging (MRI). The patients were administered an ASM following the 2012 guidelines of the National Institute for Health and Clinical Excellence (14), starting at a low dose. If the patients with NDE agreed to participate in the programme and signed an informed consent form, a baseline file was completed, which contained demographic, symptomatic and etiologic data, as well as the results of a systematic physical examination, an EEG, and an MRI. The symptomatic data were collected by interviews with the patients or the witnesses to seizure. Participants were then asked to complete a series of scales, including the Montreal cognitive assessment (MOCA), the Generalized Anxiety Disorder 7-item Scale (GAD-7), and the Chinese version of the Neurological Disorders Depression Inventory for Epilepsy (c-NDDI-E), to estimate their cognitive function and mood.

The patients enrolled in the programme were called back for a follow-up visit for treatment adjustments at 1,3 , and 6 months following the treatment and every 6 months thereafter. In cases of seizure recurrence between scheduled appointments, the patient could visit the specialist epilepsy clinics. The second ASM was considered when the first one was ineffective or the patient had intolerable side effects. At every scheduled visit, a follow-up file was completed for all patients, which recorded the patients' seizure types and frequency, the doses of the ASMs administered, and any adverse effects. If a face-to-face visit was inconvenient, the follow-up file would be completed by physicians based on the interviews with patients or caregivers by telephone. Instances of patients withdrawing the ASMs without medical advice were defined as poor compliance. Patients were excluded if the followup periods were $<12$ months. The ASMs were gradually reduced and stopped if the patients had no breakthrough seizure for at least 3 years and the repeated EEG was normal.

\section{Statistical Analyses}

Student's $t$-test, analysis of variance (ANOVA), Pearson's chisquared test, the rank-sum test, and Fisher's exact test were used to compare continuous and categorical variables. A survival (Kaplan-Meier) analysis was often used to visually summarize time-to-event data and Log-rank was used to estimate the difference between the groups. Cox regression model analysis was applied to identify the risk factors for retention of the first ASM. Logistic regression was used to analyse the risk factors of RE.

Values for continuous variables are expressed as mean \pm standard deviation (SD), and values for categorical variables are expressed as frequencies (\%). All $p$-values were from two-tailed tests. $P<0.05$ was considered to indicate statistical significance. The data were inputted by EpiData software (The EpiData Association, Odense, Denmark) and were subsequently analyzed using SPSS for Windows, Version 24.0 (SPSS Inc., Chicago, IL, USA).

\section{Ethical Approval}

The protocol for this study was approved by the Ethics Committee of the First Hospital of Jilin University [the approval number: 2017-326] and was performed in accordance with the ethical standards laid down in the 1964 Declaration of Helsinki and its later amendments. Each enrolled patient provided a signed informed consent form before the study began.

\section{RESULT}

\section{Demographic Information}

A total of 6,636 people with epilepsy (PWE) who visited the Epilepsy Diagnosis and Treatment Center of the First Hospital of Jilin University were screened, and 466 patients were diagnosed as NDE and enrolled in the programme. The demographic 
TABLE 1 | Demographic data of the patients with newly diagnosed epilepsy, $N$ (\%) or mean \pm standard deviation.

\begin{tabular}{|c|c|c|c|c|}
\hline Variable & $\begin{array}{l}\text { Total at start } \\
\qquad(n=466)\end{array}$ & $\begin{array}{l}\text { Refractory epilepsy } \\
\qquad(n=68)\end{array}$ & $\begin{array}{l}\text { Seizure free } \\
\qquad(n=244)\end{array}$ & $P$-value ${ }^{d}$ \\
\hline Gender & & & & 0.142 \\
\hline Male & $283(61)$ & $46(68)$ & $141(58)$ & \\
\hline Female & $183(39)$ & $22(32)$ & $103(42)$ & \\
\hline Age of onset, y & $31.2 \pm 18.5$ & $27.4 \pm 16.7$ & $31.4 \pm 18.3$ & 0.093 \\
\hline Duration of disease, y & $3.91 \pm 7.69$ & $4.75 \pm 8.75$ & $3.42 \pm 7.10$ & 0.280 \\
\hline $\begin{array}{l}\text { Baseline frequency of seizure per } \\
\text { month, median (interquartile range) }\end{array}$ & $1.00(2.52)$ & $2.75(14.0)$ & $1.00(1.50)$ & $<0.001$ \\
\hline $\begin{array}{l}\text { Lower average income }(<160 \\
\text { USD/month) }\end{array}$ & $61(13)$ & $14(21)$ & $27(11)$ & 0.040 \\
\hline Types of seizure & & & & 0.089 \\
\hline Focal & $418(90)$ & $66(97)$ & $215(88)$ & \\
\hline Generalized & $43(9.2)$ & $2(2.9)$ & $26(11)$ & \\
\hline Unknown & $5(1.1)$ & $0(0.0)$ & $3(1.2)$ & \\
\hline History of status epilepticus & $21(4.5)$ & $5(7.4)$ & 8 (3.3) & 0.137 \\
\hline Etiology & & & & 0.212 \\
\hline Structural & $96(21)$ & $19(28)$ & $41(17)$ & \\
\hline Genetic & $1(0.2)$ & $0(0.0)$ & $1(0.4)$ & \\
\hline Infectious & $9(1.9)$ & $1(1.5)$ & $5(2.0)$ & \\
\hline Immune & $1(0.2)$ & $0(0.0)$ & $0(0.0)$ & \\
\hline Unknown & $359(77)$ & $48(71)$ & $197(80)$ & \\
\hline Family history of epilepsy & $49(11)$ & $11(16)$ & $24(9.8)$ & 0.143 \\
\hline History of febrile seizure & $44(9.4)$ & $7(10.3)$ & $23(9.4)$ & 0.671 \\
\hline $\mathrm{MOCA}^{\mathrm{a}+}$, score & $24.1 \pm 4.62$ & $24.1 \pm 4.58$ & $24.6 \pm 4.24$ & 0.482 \\
\hline GAD- $7^{b}$, score & $4.62 \pm 4.38$ & $5.35 \pm 4.76$ & $4.58 \pm 4.05$ & 0.428 \\
\hline c-NDDI-E' , score & $8.09 \pm 3.19$ & $8.34 \pm 3.34$ & $7.90 \pm 3.02$ & 0.444 \\
\hline
\end{tabular}

aMOCA, Montreal cognitive assessment.

${ }^{b} \mathrm{GAD}-7$, Generalized Anxiety Disorder 7-item Scale.

${ }^{C} C-N D D I-E$, Chinese version of the Neurological Disorders Depression Inventory for Epilepsy.

${ }^{d}$ The $p$-value between the refractory group and seizure-free group.

information is shown in Table 1. The median follow-up time was 24 (range, 12-48) months. After treatment adjustments based on the responses to ASMs, $52 \%(n=244)$ of the patients achieved seizure-free status; however, $15 \%(n=68)$ were diagnosed as RE. The others $(33 \%, n=154)$ were undetermined (Figure 1). The median duration of treatment before arriving at RE and seizurefree status were 12 (range, 3-36) months and 12 (range, 12-36) months, respectively. About $74 \%(n=50)$ of the patients required at least 12 months before being diagnosed with RE.

Comparing the demographic data between the RE group and the seizure-free group, patients with RE were inclined to having a lower average income $(Z=-1.764, p=0.078)$ and younger age of onset $(Z=-1.679, p=0.093)$. The baseline seizure frequency in the RE group was more than that in the seizure-free group $(Z$ $=-3.911, p<0.001)$.

\section{Response to the First ASM}

The first ASMs administrated to the patients are shown in Table 2. The focal seizure was the most common type of seizure and oxcarbazepine was the most commonly used ASM. A total of $370(79 \%)$ patients remained on the first ASM at the last follow-up and 286 (61\%) patients remained on the first ASM as monotherapy, among which 186 (40\%) patients achieved seizure-free status. Among those who did not reach seizurefree status with the first ASM, 174 patients were treated with monotherapy (100 remaining on the first ASM with increased dosage and 74 switching to another monotherapy) and 102 patients with multiple therapy at the last visit; among these patients, $24 \%(n=68)$ developed RE and $21 \%(n=58)$ were seizure-free. For those who reached seizure-free status with the first ASM, the maintenance doses are shown in Table 2. The median maintenance doses were no more than 50\% DDD except for oxcarbazepine. At the 12- and 24-month followup, lamotrigine (88 and $82 \%$ ), levetiracetam (82 and 82\%), and oxcarbazepine ( 84 and $83 \%$ ) had a higher probability of retention, and topiramate had the lowest probability of retention (56 and 56\%, respectively). Carbamazepine, phenobarbital, and other ASM (pregabalin and gabapentin) were excluded from the comparison due to the limited number of patients. The probability of retention of the first ASM is shown in Figure 2. There was a significant difference between the probability of the different types of ASMs $\left(\chi^{2}=17.807, p=0.001\right)$. A total of $183(39 \%)$ patients reduced the dose of the first ASM due to adverse effects, among whom 96 patients withdrew the 


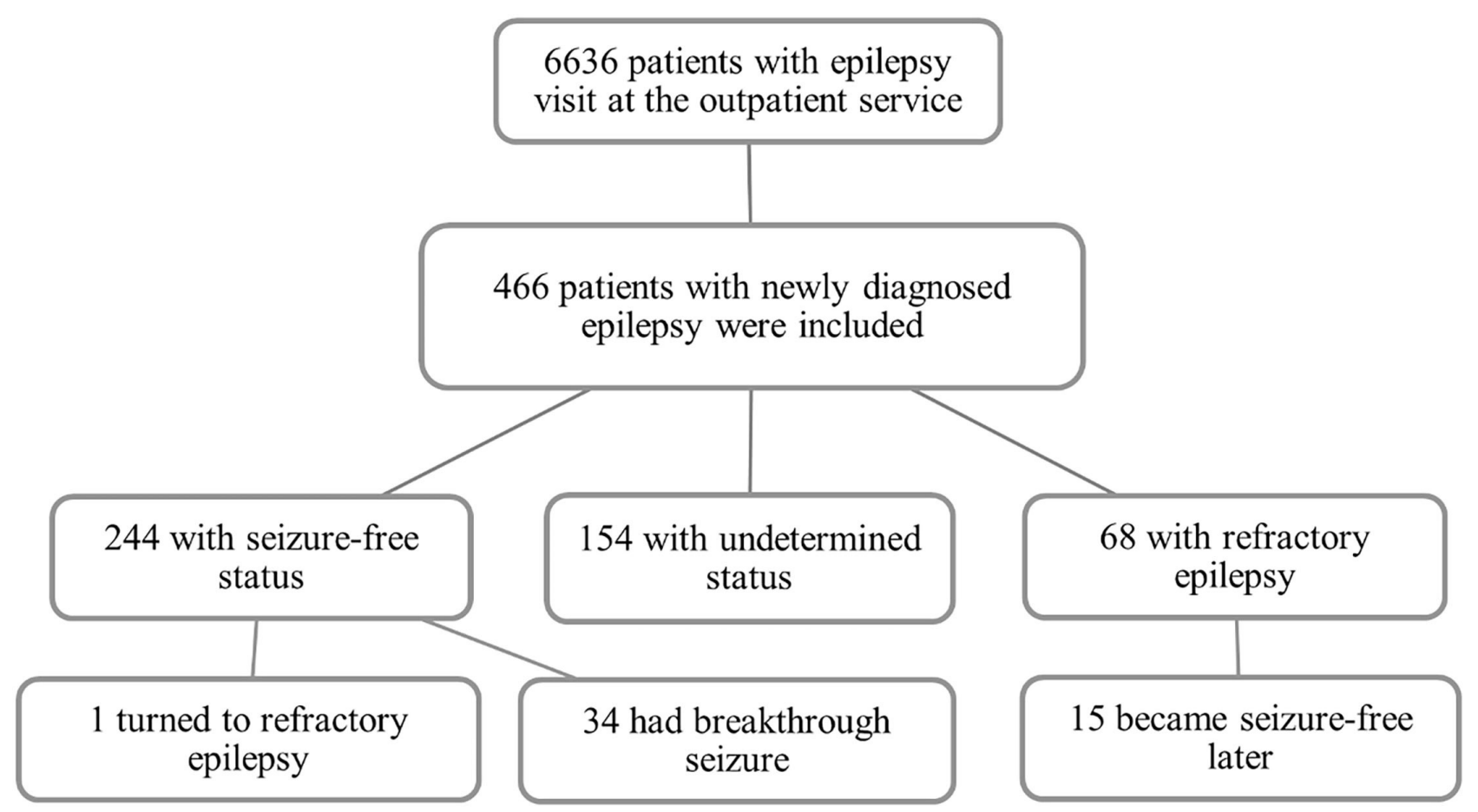

FIGURE 1 | Flow diagram of the study.

first ASM. The causes of withdrawal or dose-reduction are shown in Table 3. The objective adverse effects were drowsiness, ataxia, dizziness, headache, memory decline, irritability, weight gain or loss, palpitation, and gastrointestinal complaints, among others.

Cox regression was used to analyse the influencing factors of the retention of the first ASM. Considering the types of the first ASM, gender, age of onset, average income, disease duration, seizure frequency, and types of seizure at baseline as independent variables in the Cox regression model analysis of the first ASM retention, the hazard ratio (HR) of withdrawal of valproic acid and topiramate were 2.31 [95\% confidence intervals (CI): $1.35-$ 3.93] and 2.93 (95\% CI: 1.38-6.20), respectively, compared to that of oxcarbazepine.

\section{Risk Factors of Refractory Epilepsy}

At the last visit, $4(0.9 \%)$ patients were receiving no ASM, and $360(77 \%), 81(17 \%), 18(4 \%)$, and $3(0.6 \%)$ patients were receiving one, two, three, and four ASMs, respectively. The ratio of seizure-free patients was $0.4 \%$ (no ASM, $n=1$ ), $91 \%$ (one ASM, $n=223$ ), $7.4 \%$ (two ASMs, $n=18$ ), and $0.8 \%$ (three ASMs, $n=2$ ), respectively. During the treatment, 83 (18\%) patients had ever withdrawn the ASMs without medical advice but the ASMs were re-administered at the nearest follow-up. Approximately 24\% $(n=16)$ of the patients in the RE group and $16 \%(n=38)$ in the seizure-free group had poor compliance, and no significant difference was found $\left(\chi^{2}=2.352, p>0.05\right)$. Breakthrough seizures during the first expected interictal interval following ASM treatment were compared between the RE $(77 \%, n=52)$ and seizure-free group $(26 \%, n=63)$, and there was a significant difference $\left(\chi^{2}=58.622, p<0.01\right)$.

Fifteen (22\%) patients who had been diagnosed with RE reached seizure-free status following ASM adjustment (they were still classified to RE in the statistics described above). Among them, six, eight, and one patient(s) were treated with one, two, and three $\operatorname{ASM}(\mathrm{s})$, respectively. No significant difference in the demographic data was found between the patients with $\mathrm{RE}$ who achieved seizure-free status and patients who had not $(p>0.05)$. Finding alternative effective ASMs and increasing doses of the ASM in use were methods to achieve seizure-free status. Thirty-four patients (14\%) had breakthrough seizures after being classified as seizure-free. The time of relapse was 6 to 36 months (median 6 months) (Figure 3). No significant difference in the demographic data or poor compliance was found between patients with seizure relapse and those without $(p>0.05)$. One patient developed RE after identifying as seizure-free.

Logistic regression was applied to analyse the risk factors of $\mathrm{RE}$, and gender, age of onset, average income, disease duration, seizure frequency and types of seizure at baseline, history of status epilepticus, etiology, compliance, and breakthrough seizures during the first expected interictal interval were set as independent variables (Table 4). Breakthrough seizures during the first expected interictal interval $(\mathrm{OR}=5.66,95 \% \mathrm{CI}$ : 3.05-10.51) and higher seizure frequency (increased every 5 times/month) (OR $=1.20,95 \% \mathrm{CI}: 1.02-1.41)$ were risk factors. When the scores of MOCA, GAD-7, and c-NDDI-E were 
TABLE 2 | Doses of the first antiseizure medication (ASM) for patients who reached seizure-free status with the first ASM.

\begin{tabular}{|c|c|c|c|c|c|c|}
\hline & $\begin{array}{c}\text { At baseline, } \\
n(\%)\end{array}$ & $\begin{array}{l}\text { As the only } \\
\text { monotherapy, } n(\%)\end{array}$ & $\begin{array}{l}\text { Seizure-free, } \\
n(\%)\end{array}$ & $\begin{array}{l}\text { Median, } \\
\mathrm{mg} / \mathrm{d}\end{array}$ & $\begin{array}{l}\text { Maximum, } \\
\mathrm{mg} / \mathrm{d}\end{array}$ & $\begin{array}{c}\text { Minimum, } \\
\text { mg/d }\end{array}$ \\
\hline Valproic acid & $52(11)$ & $20(7.0)$ & $14(70)$ & 500.0 & 750 & 400 \\
\hline Carbamazepine & $8(1.7)$ & $4(1.4)$ & $2(50)$ & 500.0 & 800 & 200 \\
\hline Oxcarbazepine & $279(60)$ & $186(65)$ & $122(66)$ & 600.0 & 1,200 & 240 \\
\hline Topiramate & $19(4.1)$ & $5(1.7)$ & $2(40)$ & 100.0 & 125 & 50 \\
\hline Levetiracetam & $78(16.7)$ & $52(18)$ & $37(71)$ & 750.0 & 1,250 & 375 \\
\hline Phenobarbital & $4(0.9)$ & $3(1.0)$ & $1(33)$ & - & - & - \\
\hline Lamotrigine & $24(5.2)$ & $17(6.0)$ & $8(47)$ & 112.5 & 150 & 100 \\
\hline Others $^{a}$ & $2(0.4)$ & $1(0.3)$ & $0(0.0)$ & - & - & - \\
\hline
\end{tabular}

a "Others" refers to pregabalin and gabapentin.

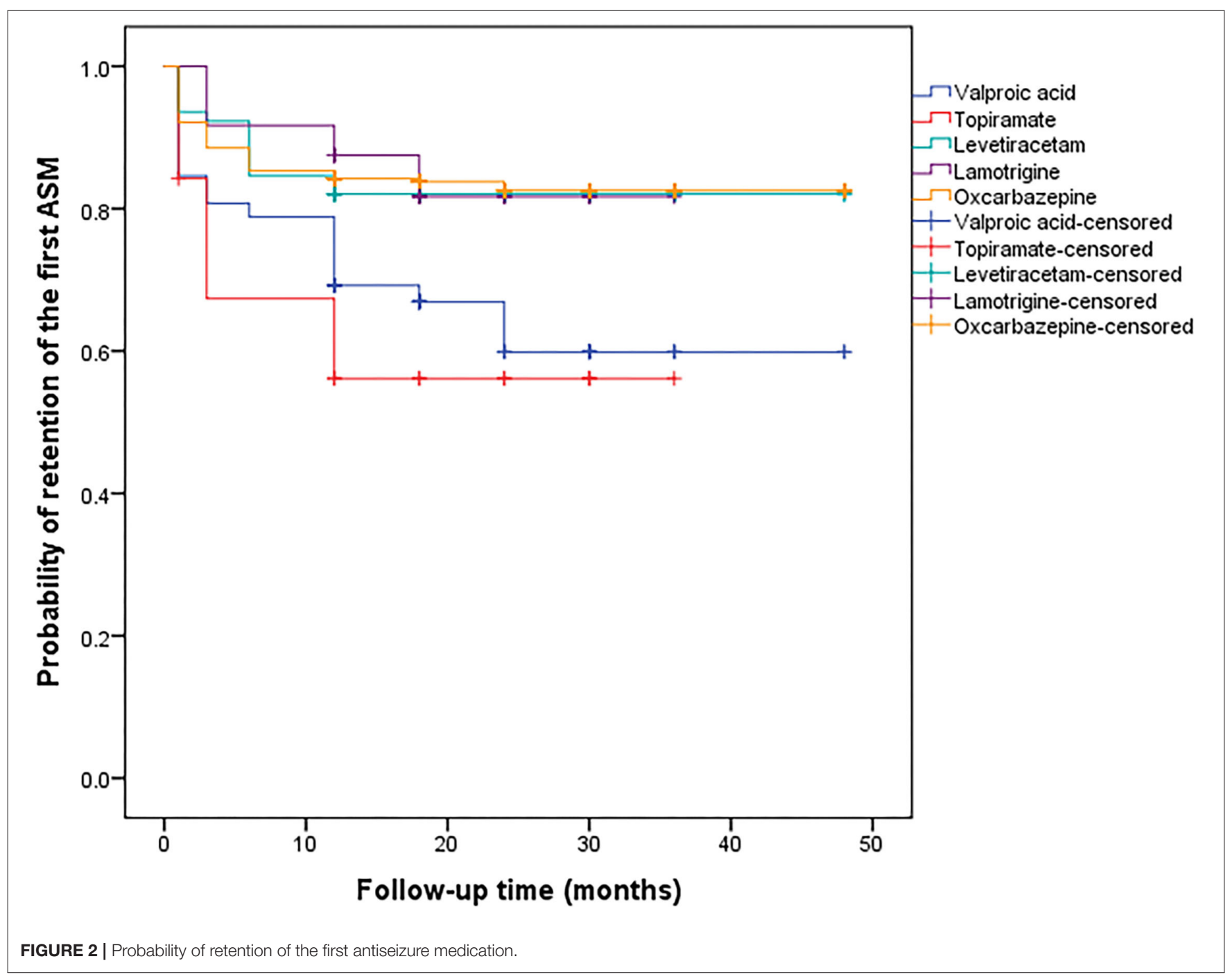

adjusted in the analysis, men were more likely to develop $\mathrm{RE}$ than women [Odds ratio $(\mathrm{OR})=2.66$, 95\% CI: $1.26-$ $5.62]$, and a younger age of onset (decrease of every 10 years) $(\mathrm{OR}=1.42,95 \% \mathrm{CI}: 1.12-1.79)$ was also a risk factor of
RE. Meanwhile, the ORs of breakthrough seizure during the first expected interictal interval and higher seizure frequency were 5.53 (95\% CI: 2.57-11.92) and 1.22 (95\% CI: 1.02-1.46), respectively (Table 4 ). 
TABLE 3 | Causes of withdrawal or dose-reduction of the first antiseizure medication, $n$ (\%).

\begin{tabular}{|c|c|c|c|c|c|c|}
\hline & Allergy & Ineffective & Liver damage & $\begin{array}{l}\text { Other objective adverse } \\
\text { effects }\end{array}$ & Poor compliance & Seizure-free for $3 y$ \\
\hline Valproic acid & $3(9.4)$ & $9(28)$ & $4(13)$ & $9(28)$ & $8(19)$ & $1(3.1)$ \\
\hline Carbamazepine & $0(0.0)$ & $0(0.0)$ & $1(25)$ & $1(25)$ & $2(50)$ & $0(0.0)$ \\
\hline Oxcarbazepine & $19(19)$ & $16(16)$ & $24(25)$ & $28(29)$ & $10(10)$ & $1(1.0)$ \\
\hline Topiramate & $3(21)$ & $2(14)$ & $0(0.0)$ & $6(43)$ & $3(21)$ & $0(0.0)$ \\
\hline Levetiracetam & $1(3.8)$ & $7(26)$ & $7(26)$ & $9(33)$ & $2(7.4)$ & $1(3.7)$ \\
\hline Phenobarbital & $0(0.0)$ & $1(50)$ & $1(50)$ & $0(0.0)$ & $0(0.0)$ & $0(0.0)$ \\
\hline Lamotrigine & $1(17)$ & $3(50)$ & $1(17)$ & $0(0.0)$ & $1(17)$ & $0(0.0)$ \\
\hline Others $^{a}$ & $0(0.0)$ & $0(0.0)$ & $0(0.0)$ & $1(100)$ & $0(0.0)$ & $0(0.0)$ \\
\hline
\end{tabular}

a "Others" refers to pregabalin and gabapentin.

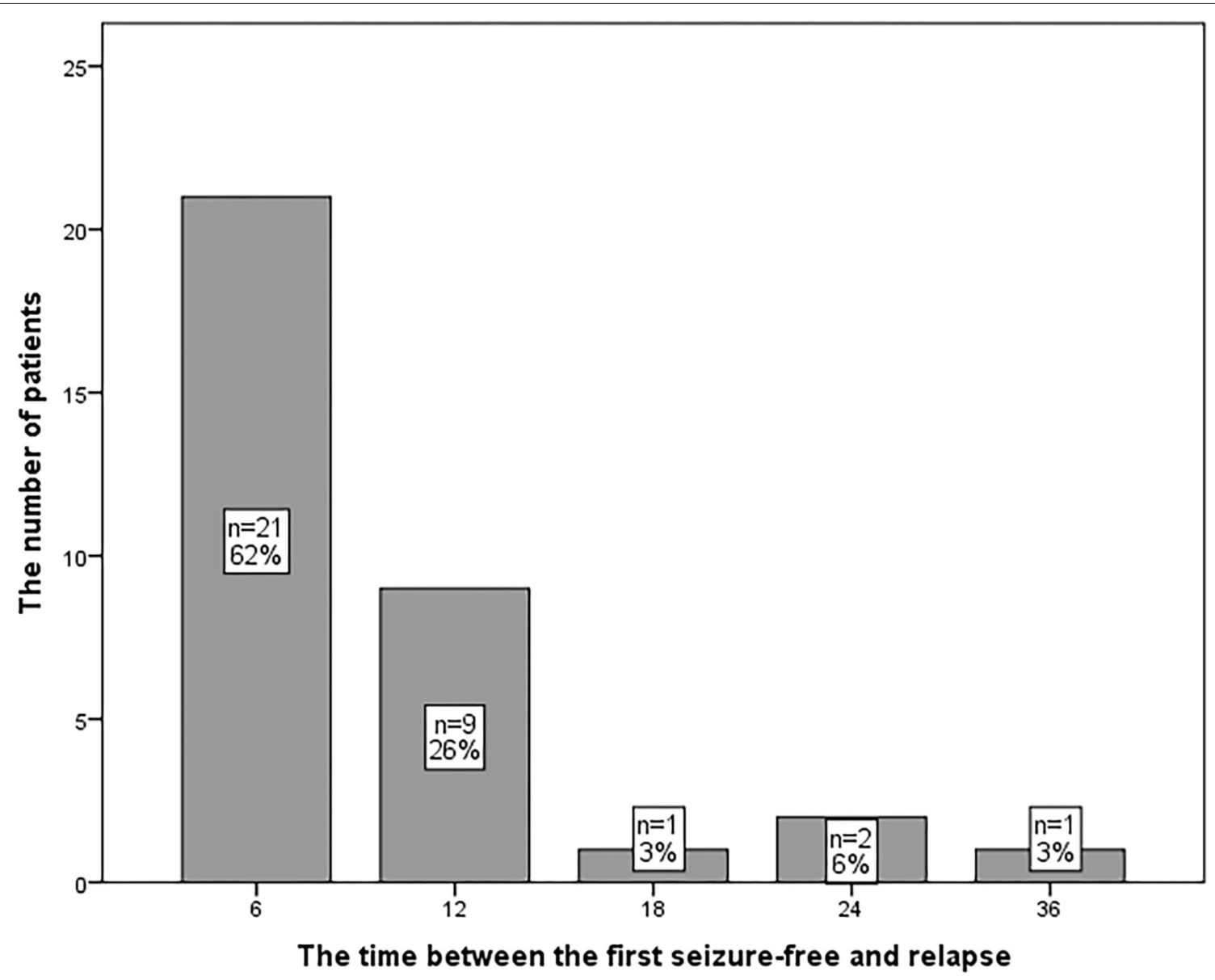

FIGURE 3 | The distribution of patients with seizure relapse.

\section{DISCUSSION}

ILAE published a new definition of RE in 2010 to set up explicit and practical criteria. Based on this definition, we conducted the first prospective study on treatment outcome of NDE in Northeast China, and we identified the risk factors of RE according to the new definition, which can help physicians more quickly and accurately identify patients that are likely to develop RE.

Nearly half of the adult patients with NDE became seizure-free in our study and $91 \%$ of them were treated with monotherapy. This proportion is lower than that in previous studies $(15,16)$, but the criteria in these studies were relatively lenient compared to the ILAE criteria (no seizures for at least the previous year). Forty 
TABLE 4 | The logistic regression analysis for risk factors of refractory epilepsy in newly diagnosed epilepsy.

\begin{tabular}{|c|c|c|c|c|c|}
\hline & \multirow[t]{2}{*}{ Variables } & \multirow[t]{2}{*}{$p$-value } & \multirow[t]{2}{*}{ OR } & \multicolumn{2}{|c|}{$95 \% \mathrm{Cl}$} \\
\hline & & & & Lower & Upper \\
\hline \multirow[t]{2}{*}{$\begin{array}{l}\text { Before adjusted by } \\
\text { scales }\end{array}$} & $\begin{array}{l}\text { Breakthrough seizures during the first expected interictal } \\
\text { interval }\end{array}$ & $<0.001$ & 5.66 & 3.05 & 10.51 \\
\hline & Higher seizure frequency (increased every 5 times/month) & 0.033 & 1.20 & 1.02 & 1.41 \\
\hline \multirow[t]{4}{*}{ After adjusted by scales } & $\begin{array}{l}\text { Breakthrough seizures during the first expected interictal } \\
\text { interval }\end{array}$ & $<0.001$ & 5.53 & 2.57 & 11.92 \\
\hline & Higher seizure frequency (increased every 5 times/month) & 0.033 & 1.22 & 1.02 & 1.46 \\
\hline & Male gender & 0.010 & 2.66 & 1.26 & 5.62 \\
\hline & Younger age of onset (decrease of every 10 years) & 0.003 & 1.42 & 1.12 & 1.79 \\
\hline
\end{tabular}

percent of the patients achieved seizure-free status with the first monotherapy and the median maintenance doses were no more than $50 \%$ DDD except for oxcarbazepine. This is consistent with the conclusion of previous studies that responsiveness may be identified with exposure to low ASM doses $(12,17)$. Most of the seizure-free statuses were obtained by monotherapy. Although Chi et al. found that combination therapy could increase the ratio of seizure-free patients compared to monotherapy (18), the latter is more acceptable for PWE in our clinic for fear of adverse effects. Dash et al. also found that reduction of the numbers of ASM may not aggravate seizures but decrease the side effects (19). Hence, combination therapy was always applied during the period of switching to another ASM or when the monotherapy did not work in our experience.

The probability of retention and the efficacy of levetiracetam and oxcarbazepine were satisfactory as the monotherapy, and liver damage and other objective adverse effects were the main causes of withdrawal. As a traditional ASM, valproic acid had relatively lower retention but it was also very efficient. Lamotrigine had a high likelihood of retention but did not perform as well as the other drugs. Neither the retention nor the efficacy of topiramate were satisfactory, and objective adverse effects were the main cause of withdrawal. In some studies with children, lamotrigine had better retention than oxcarbazepine (20) and topiramate (21). For older adults, carbamazepine is more likely to cause withdrawal symptoms than lamotrigine, levetiracetam, and valproic acid (22). Levetiracetam, on the other hand, has better efficacy than that of lamotrigine (23). Levetiracetam and oxcarbazepine were the more favorable drugs in terms of better tolerance and efficacy in our study. Unfortunately, we could not analyse their retention in older adults due to the limited number of patients.

The incidence of RE in adult NDE in our study was 15\%, which is similar to the result of the systematic review on NDE (17\%) (24). Although the ILAE definition is the minimum criteria, it could take more than 1 year for the majority of the patients to identify as RE. Moreover, patients with RE were inclined to have lower income, which means that the pharmacotherapy with the possibility of poor effect would put a huge burden on this population. Timely diagnosis helps physicians and patients to consider other optimal treatments, such as resective or palliative surgery, neurostimulation $(25,26)$, and ketogenic diet $(27)$.
Breakthrough seizures during the first expected interictal interval, high seizure frequency at baseline, younger age of onset, and the male sex were risk factors of RE in our study. Younger age at seizure onset and high initial seizure frequency were discussed as predictors of RE in previous studies (28-30). The breakthrough seizures during the first expected interictal interval reflect responses to the first ASM and the longitudinal data could be a more accurate predictor. Jiang et al. posited that more than two seizures in the first year after ASM initiation predicted less likelihood of achieving 2-year remission. Making the interictal interval as the observing time may be more suitable for each PWE with different seizure periods. Hughes et al. (31) found both the presence and number of post-breakthrough seizures indicated poor outcomes. Only one patient developed RE after achieving seizure-free status in our study, and others were undetermined for limited post-seizure follow-up; therefore we cannot reach the same conclusion. Previous research found that men were more susceptible to temporal lobe epilepsy-like seizures and seizurerelated damage (32). Therefore, the severity of epilepsy and the degree of hemicranial volume loss were worse in men than that in women. The finding supports our conclusion that male sex was a risk factor of RE.

Nearly $14 \%$ of the patients with seizure-free status had seizure relapse and $88 \%$ of them had a relapse within 12 months. Hence, prolonging the period of ASM treatment and careful withdrawal should be emphasized, and the minimum period of ASM treatment should be 2 years of seizure-free status (33). Although diagnosing as RE, $22 \%$ of the patients achieved seizurefree status after changing to the alternative ASM regimen or increasing the doses of the ASMs in use, which is supported by a previous study (34). A patient with identified seizure-free status developed RE later in the course of her epilepsy. This is consistent with the patterns of previous research, and excessive expression of transporters for ASM removal and reduced drugtarget sensitivity are the major probable theories (35). A new approach in anti-epilepsy rather than antiseizure treatment is necessary to reverse the unsatisfactory treatment scenario.

In conclusion, treatment outcomes of the majority of the NDE are good, and monotherapy could be efficient at a low dose. Levetiracetam and oxcarbazepine performed best in tolerance and efficacy. Breakthrough seizures during the first expected interictal interval, high seizure frequency at baseline, younger 
age of onset, and male sex predicted RE. Achieving seizure-free status is not enough to start the withdrawal of ASMs. RE is not permanent and seizure-free may be achieved subsequently by appropriate drug adjustment.

\section{LIMITATION}

This was a single-center study and the findings might be difficult to extrapolate in the global settings. The follow-up period was not sufficient to determine RE for a part of patients. However, as our program is still going on, the follow-up time would be extended and the "undetermined" patients may achieve their outcome at the subsequent visits.

\section{DATA AVAILABILITY STATEMENT}

The raw data supporting the conclusions of this article will be made available by the authors, without undue reservation.

\section{ETHICS STATEMENT}

The studies involving human participants were reviewed and approved by the Ethics Committee of The First Hospital of Jilin University [the approval number: 2017-326]. Written informed

\section{REFERENCES}

1. Thijs RD, Surges R, O’Brien TJ, Sander JW. Epilepsy in adults. Lancet. (2019) 393:689-701. doi: 10.1016/S0140-6736(18)32596-0

2. Song P, Liu Y, Yu X, Wu J, Poon AN, Demaio A, et al. Prevalence of epilepsy in China between 1990 and 2015: a systematic review and meta-analysis. J Glob Health. (2017) 7:020706. doi: 10.7189/jogh.07.020706

3. Mohanraj R, Brodie MJ. Early predictors of outcome in newly diagnosed epilepsy. Seizure. (2013) 22:333-44. doi: 10.1016/j.seizure.2013.02.002

4. Kwan P, Brodie MJ. Early identification of refractory epilepsy. $N$ Engl J Med. (2000) 342:314-9. doi: 10.1056/NEJM200002033420503

5. Geerts A, Arts WF, Stroink H, Peeters E, Brouwer O, Peters B, et al. Course and outcome of childhood epilepsy: a 15-year follow-up of the Dutch Study of Epilepsy in Childhood. Epilepsia. (2010) 51:118997. doi: 10.1111/j.1528-1167.2010.02546.x

6. Huang L, Li S, He D, Bao W, Li L. A predictive risk model for medical intractability in epilepsy. Epilepsy Behav. (2014) 37:282-6. doi: 10.1016/j.yebeh.2014.07.002

7. Aaberg KM, Bakken IJ, Lossius MI, Lund Søraas C, Tallur KK, Stoltenberg C, et al. Short-term seizure outcomes in childhood epilepsy. Pediatrics. (2018) 141:e20174016. doi: 10.1542/peds.2017-4016

8. Kwan P, Arzimanoglou A, Berg AT, Brodie MJ, Hauser WA, Mathern G, et al. Definition of drug resistant epilepsy: consensus proposal by the ad hoc Task Force of the ILAE Commission on Therapeutic Strategies. Epilepsia. (2010) 51:1069-77. doi: 10.1111/j.1528-1167.2009.02397.x

9. Fisher RS, Acevedo C, Arzimanoglou A, Bogacz A, Cross JH, Elger CE, et al. ILAE official report: a practical clinical definition of epilepsy. Epilepsia. (2014) 55:475-82. doi: 10.1111/epi.12550

10. Scheffer IE, Berkovic S, Capovilla G, Connolly MB, French J, Guilhota L, et al. ILAE classification of the epilepsies: position paper of the ILAE Commission for Classification and Terminology. Epilepsia. (2017) 58:51221. doi: 10.1111/epi.13709

11. Proposal for revised classification of epilepsies and epileptic syndromes. Commission on Classification and Terminology of the International League Against Epilepsy. Epilepsia. (1989) 30:389-99. doi: 10.1111/j.1528-1157.1989.tb05316.x consent to participate in this study was provided by the participants' legal guardian/next of kin.

\section{AUTHOR CONTRIBUTIONS}

NL, JL, CC, and YC are responsible for including participants, data entry, and following up. WL is the designer of this project. All authors contributed to the article and approved the submitted version.

\section{FUNDING}

The work was supported by fund from the Clinical Research Development Fund of The First Hospital of Jilin University [fund number: lcpyjj2017006].

\section{ACKNOWLEDGMENTS}

We thank Xin Zhang, Rui Zhong, Mengmeng Li, Yingxue Lu, and Qian Zhao for their help in including participants, data entry, and following up.
12. Brodie MJ, Barry SJ, Bamagous GA, Kwan P. Effect of dosage failed of first antiepileptic drug on subsequent outcome. Epilepsia. (2013) 54:1948. doi: 10.1111/j.1528-1167.2012.03722.x

13. French J, Friedman D. Epilepsy: from newly diagnosed to treatment-resistant disease. Lancet Neurol. (2011) 10:9-11. doi: 10.1016/S1474-4422(10)70301-X

14. National Clinical Guideline Centre. National Institute for Health and Clinical Excellence: Guidance. The Epilepsies: The Diagnosis and Management of the Epilepsies in Adults and Children in Primary and Secondary Care: Pharmacological Update of Clinical Guideline 20. London: Royal College of Physicians (2012).

15. Brodie MJ, Barry SJ, Bamagous GA, Norrie JD, Kwan P. Patterns of treatment response in newly diagnosed epilepsy. Neurology. (2012) 78:154854. doi: 10.1212/WNL.0b013e3182563b19

16. Chen Z, Brodie MJ, Liew D, Kwan P. Treatment outcomes in patients with newly diagnosed epilepsy treated with established and new antiepileptic drugs: a 30-year longitudinal cohort study. JAMA Neurol. (2018) 75:27986. doi: 10.1001/jamaneurol.2017.3949

17. Poolos NP, Castagna CE, Williams S, Miller AB, Story TJ. Association between antiepileptic drug dose and long-term response in patients with refractory epilepsy. Epilepsy Behav. (2017) 69:59-68. doi: 10.1016/j.yebeh.2016. 10.010

18. Chi X, Li R, Hao X, Chen J, Xiong W, Xu H, et al. Response to treatment schedules after the first antiepileptic drug failed. Epilepsia. (2018) 59:211824. doi: 10.1111/epi.14565

19. Dash D, Aggarwal V, Joshi R, Padma MV, Tripathi M. Effect of reduction of antiepileptic drugs in patients with drug-refractory epilepsy. Seizure. (2015) 27:25-9. doi: 10.1016/j.seizure.2015.02.025

20. Hur YJ. Comparison of lamotrigine and oxcarbazepine monotherapy for pediatric focal epilepsy: an observational study. Seizure. (2018) 60:1236. doi: 10.1016/j.seizure.2018.06.013

21. Mills JK, Lewis TG, Mughal K, Ali I, Ugur A, Whitehouse WP. Retention rate of clobazam, topiramate and lamotrigine in children with intractable epilepsies at 1 year. Seizure. (2011) 20:402-5. doi: 10.1016/j.seizure.2011.01.011

22. Lattanzi S, Trinka E, Del Giovane C, Nardone R, Silvestrini M, Brigo F. Antiepileptic drug monotherapy for epilepsy in the elderly: 
a systematic review and network meta-analysis. Epilepsia. (2019) 60:2245-54. doi: 10.1111/epi.16366

23. Lezaic N, Gore G, Josephson CB, Wiebe S, Jetté N, Keezer MR. The medical treatment of epilepsy in the elderly: a systematic review and meta-analysis. Epilepsia. (2019) 60:1325-40. doi: 10.1111/epi.16068

24. Kalilani L, Sun X, Pelgrims B, Noack-Rink M, Villanueva V. The epidemiology of drug-resistant epilepsy: a systematic review and meta-analysis. Epilepsia. (2018) 59:2179-93. doi: 10.1111/epi.14596

25. Sheng J, Liu S, Qin H, Li B, Zhang X. Drug-resistant epilepsy and surgery. Curr Neuropharmacol. (2018) 16:1728. doi: 10.2174/1570159X15666170504123316

26. Zangiabadi N, Ladino LD, Sina F, Orozco-Hernandez JP, Carter A, TellezZenteno JF. Deep brain stimulation and drug-resistant epilepsy: a review of the literature. Front Neurol. (2019) 10:601. doi: 10.3389/fneur.2019.00601

27. Yang H, Shan W, Zhu F, Wu J, Wang Q. Ketone bodies in neurological diseases: focus on neuroprotection and underlying mechanisms. Front Neurol. (2019) 10:585. doi: 10.3389/fneur.2019.00585

28. Roy PL, Ronquillo LH, Ladino LD, Tellez-Zenteno JF. Risk factors associated with drug resistant focal epilepsy in adults: a case control study. Seizure. (2019) 73:46-50. doi: 10.1016/j.seizure.2019.10.020

29. Stevelink R, Koeleman BPC, Sander JW, Jansen FE, Braun KPJ. Refractory juvenile myoclonic epilepsy: a meta-analysis of prevalence and risk factors. Eur J Neurol. (2019) 26:856-64. doi: 10.1111/ene. 13811

30. Wassenaar M, Leijten FS, Egberts TC, Moons KG, Uijl SG. Prognostic factors for medically intractable epilepsy: a systematic review. Epilepsy Res. (2013) 106:301-10. doi: 10.1016/j.eplepsyres.2013. 06.013

31. Hughes DM, Bonnett LJ, Marson AG, Garcia-Finana M. Identifying patients who will not reachieve remission after breakthrough seizures. Epilepsia. (2019) 60:774-82. doi: 10.1111/epi.14697
32. Kipnis PA, Sullivan BJ, Kadam SD. Sex-dependent signaling pathways underlying seizure susceptibility and the role of chloride cotransporters. Cells. (2019) 8:448. doi: 10.3390/cells8050448

33. Beghi E, Giussani G, Grosso S, Iudice A, La Neve A, Pisani F, et al. Withdrawal of antiepileptic drugs: guidelines of the Italian League Against Epilepsy. Epilepsia. (2013) 54(Suppl. 7):2-12. doi: 10.1111/epi.12305

34. Hao $X$, Chen $Z$, Yan $B$, Kwan $P$, Zhou D. Impact of drug manipulation on seizure freedom in adults with uncontrolled epilepsy: a prospective controlled study in rural China. CNS Drugs. (2017) 31:237-43. doi: 10.1007/s40263-016-0397-5

35. Schmidt D, Löscher W. Drug resistance in epilepsy: putative neurobiologic and clinical mechanisms. Epilepsia. (2005) 46:85877. doi: 10.1111/j.1528-1167.2005.54904.x

Conflict of Interest: The authors declare that the research was conducted in the absence of any commercial or financial relationships that could be construed as a potential conflict of interest.

Publisher's Note: All claims expressed in this article are solely those of the authors and do not necessarily represent those of their affiliated organizations, or those of the publisher, the editors and the reviewers. Any product that may be evaluated in this article, or claim that may be made by its manufacturer, is not guaranteed or endorsed by the publisher.

Copyright (C) $2021 \mathrm{Li}, \mathrm{Li}$, Chen, Chu and Lin. This is an open-access article distributed under the terms of the Creative Commons Attribution License (CC BY). The use, distribution or reproduction in other forums is permitted, provided the original author(s) and the copyright owner(s) are credited and that the original publication in this journal is cited, in accordance with accepted academic practice. No use, distribution or reproduction is permitted which does not comply with these terms. 